\title{
Invasive sino-orbital fungal infections in immunocompetent patients: a clinico-pathological study
}

\author{
Namrata Gangaram Adulkar ${ }^{1} \cdot$ Santhi Radhakrishnan ${ }^{2} \cdot$ N. Vidhya ${ }^{1} \cdot$ Usha Kim $^{1}$
}

Received: 20 August 2017 / Revised: 12 May 2018 / Accepted: 11 October 2018 / Published online: 14 February 2019

(c) The Royal College of Ophthalmologists 2019

\begin{abstract}
Objective Invasive sino-orbital fungal infections in immunocompetent patients are a rare clinical entity; the diagnosis and management of which is challenging. We present a large case series of invasive sino-orbital fungal granulomas in patients without pre-existing systemic immunocomprimising condition.

Design Retrospective case series.

Participants Twenty cases of invasive sino-orbital/naso-orbital fungal granulomas in immunocompetent individuals.

Methods We retrospectively analyzed all patients with orbital fungal granuloma who were treated at a tertiary referral eye center in South India between January 2005 and December 2012. Histopathologic confirmation of tissue invasion by fungal elements and presence of granulomatous inflammation was established in all cases included in the study.

Main outcome measures Relief of patient symptoms, resolution/no progression of disease on orbital imaging, ocular, and vision salvage were the treatment outcomes studied.

Results Twenty patients (11 male and 9 female) were studied. Mean age of patients was 47.4 years ranging from 24-65 years. Aspergillus was the causative fungus in 18 cases while 2 were cases of mucormycosis. Surgical debulking of the orbital disease was performed in 7 patients. Exenteration was performed in 2 patients to prevent spread to the CNS. Medical therapy consisted of oral itraconazole in all patients and intravenous amphotericin B was administered in 2 patients. Average duration of medical therapy required to achieve relief from symptoms was 6-8 months. Recurrences are common and longterm follow-up is essential.

Conclusions Orbital fungal infections are challenging in terms of both diagnosis and treatment. Debulking along with prolonged antifungal therapy seems to be effective in controlling the infection.
\end{abstract}

\section{Introduction}

Invasive sino-orbital fungal infections in immunocompetent patients, is an uncommon clinical entity; the diagnosis and

This study was presented at the annual meeting of Oculoplastics Association of India, Dec 2015

Supplementary information The online version of this article (https:// doi.org/10.1038/s41433-019-0358-6) contains supplementary material, which is available to authorized users.

Namrata Gangaram Adulkar namrata.adulkar@gmail.com

1 Department of Orbit, Oculoplasty \& Ocular Oncology, Aravind Eye Hospital, Madurai, India

2 Department of Ocular Pathology, Aravind Eye Hospital, Madurai, India management of which continues to remain a challenge. Fungi like aspergillus may normally colonize the paranasal sinuses and lungs. Also a large numbers of spores are present in inhaled air. Under appropriate conditions, the spores may become saprophytic within a host and multiply. Locally aggressive, invasive fungal masses may develop, particularly if the host immunity is severely compromised. Due to close proximity of the paranasal sinuses to the orbit, contiguous spread occurs, especially in immunocompromised hosts. In immunocompetent hosts, fungal colonization usually has a chronic, slowly progressive course. It is potentially fatal due to spread to middle cranial fossa via superior orbital fissure and optic canal; with mortality of upto $80 \%$ [1]. Orbital fungal disease is not often suspected, because of its relative rarity and the wide range of orbital pathologies that can present similarly. Currently, there are no standard guidelines for management and outcome is invariably poor without timely diagnosis and appropriate treatment. 
We present a large case series of invasive sino-orbital/ naso-orbital fungal granulomas in immunocompetent subjects. The clinical presentations, imaging features, treatment, and outcomes are discussed and factors associated with visual compromise are analyzed.

\section{Materials \& methods}

A retrospective review of records of 20 patients with invasive orbital/sino-orbital fungal infection who were treated at tertiary referral eye center in South India, between January 2005 and December 2012 was done. All patients had invasive fungal elements on histopathological examination of orbital biopsy specimen, undertaken either by endoscopic or open technique.

Patient details were collected and analyzed for demographic data, clinical presentation, imaging features, treatment, and outcomes. Complete blood counts, liver and renal function tests, chest x-ray, blood glucose levels, and human immunodeficiency virus serology were noted for all patients.

Surgical treatment included debridement of the orbital granuloma and radical extirpation of all involved paranasal sinuses. The extent of orbital and paranasal sinus debridement depended on the extent of the disease, the functional status of the eye and the perceived response to medical therapy. Exenteration was performed in two patients. The final decision was taken in consultation with the patient after an informed discussion with regard to the nature of disease, its potential for intracranial spread and for a fatal outcome, and the relative benefits and hazards of a conservative orbital debridement versus a radical orbital exenteration.

The surgically excised pathological orbital specimen, whether obtained by biopsy procedures, or radical clearance; performed by endoscopic or open route, was subject to a standard fixation protocol and the sections were stained with haematoxylin and eosin ( $\mathrm{H}$ and $\mathrm{E})$, para-amino salicylic, and Gomori's methenamine silver (GMS) stains.

Institutional ethics committee approval was obtained prior to commencing the study.

\section{Results}

\section{Demographics}

There were 12 males and 8 females, mean age in the study cohort was 47.4 years (range 24-65 years). Right orbit was involved more commonly involved and right sided disease was seen in 11 patients.

\section{Presentations}

Proptosis was the most common presenting complaint and was seen in 15 patients, followed by diplopia in 5 patients and diminution of vision in 4 patients. Proptosis (15 patients) was also the most common sign on examination followed by limitation of ocular motility ( 9 patients). Two patients presented with discharging periocular sinuses and 2 presented with palpable mass lesion in the medial canthal region. [Fig. 1]

Mean duration of complaints before presentation was 6.05 months (range 2 weeks to 36 months). Most common diagnosis prior to presentation was idiopathic orbital inflammatory disease (7 patients), 1 each of orbital cellulitis, rhinosporidiosis, tuberculosis, tolosa hunt syndrome, and orbital foreign body. One patient was diagnosed of basal cell carcinoma at the medial canthus and referred for further management. One patient presented with perforated fungal corneal ulcer and underwent evisceration for fungal
Fig. 1 Clinical presentations. a swelling near right medial canthus, b right eye dystopia, $\mathbf{c}$ right eye axial proptosis, $\mathbf{d}$ left orbital apex syndrome

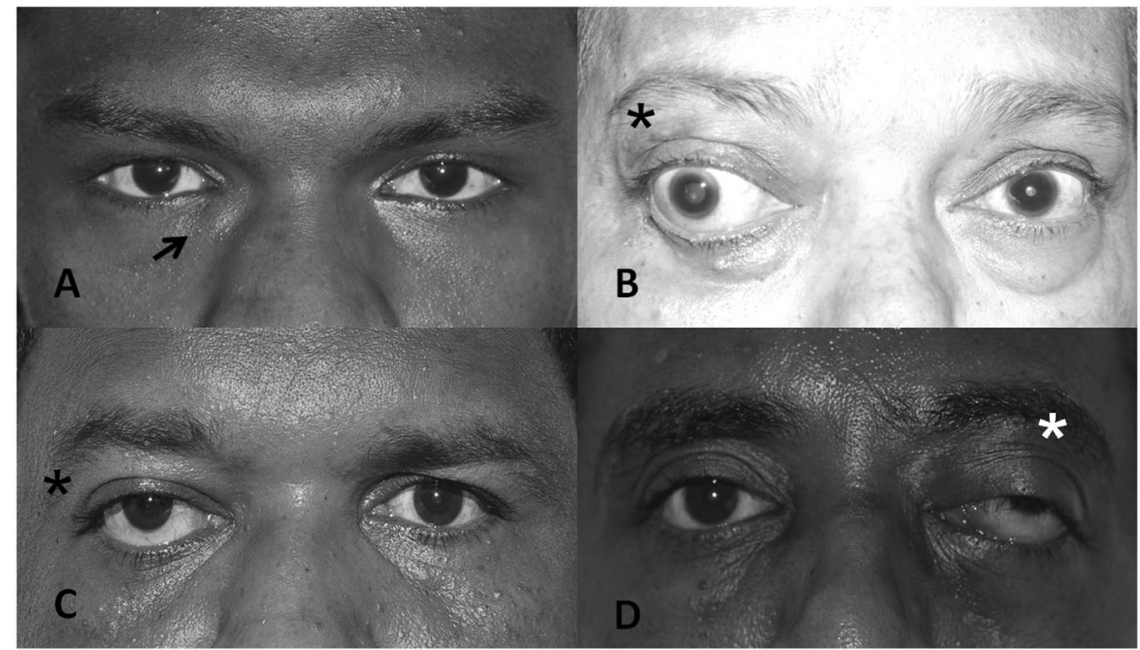


endophthalmitis. Four months after evisceration, she presented with a mass in the anophthalmic socket which was then exenterated. Only 2 of the 20 patients, were already diagnosed cases of sino-orbital fungal disease and were receiving antifungal drugs when they presented to us. Four patients presented to us primarily, with orbital symptoms whereas rest of the patients had been seen by an ophthalmologist before they presented to our clinic. Ten patients received systemic steroids; including intravenous steroids followed by oral steroids in 4 and oral steroids alone in 6 patients.

\section{Imaging findings}

Computed tomography (CT) was most commonly performed imaging study in our series. On CT scan of orbit, most patients presented with an ill-defined, heterogeneous, hyperdense mass or infiltration of orbit, and stranding of orbital fat as seen in idiopathic orbital inflammatory disease.

In three patients, the pathology was primarily preseptal with secondary infiltration into orbital fat, whereas 17 patients presented with orbital (intraconal and extraconal) with infiltration of the extraocular muscles. Lateral rectus, inferior rectus, and inferior oblique were most commonly involved. The disease process reached orbital apex in 7 patients; and into the infratemporal fossa in 4 patients. Bone erosion was present in 10 patients. Pansinusitis was present in 14 patients with ethmoidal sinuses most commonly involved. [Fig. 2] On magnetic resonance imaging (MRI), the lesions were isointense to muscle on T1-weighted and hypointense on T2-weighted images.

\section{Histopathology}

Histopathologic examination showed tissue invasion by fungal hyphae, with characteristics of Aspergillus in 18 patients: non-caseating multinucleate giant cell granulomas, fibrosis and chronic inflammatory infiltrate composed of eosinophils, lymphocytes, and plasma cells in 18 cases. Aspergillus hyphae were identified by their characteristic uniform size, dichotomous branching approximately at 45 degrees, and presence of septations that were better seen with Gomori's silver methenamine stain than with hematoxylin- eosin. [Fig. 3] Two patients showed presence of mucor colonies in the orbital tissue with characteristic angio-invasion. [Fig. 4] Mucor was identified by the presence of flat, elongated, ribbon like, collapsed aseptate fungal hyphae with obtuse angled branching patterns. [Fig. 4]

\section{Medical treatment}

All patients received oral itraconazole after reviewing baseline liver function parameters. Patients received itraconazole in the dosage of 200-400 mg daily (divided doses) for duration ranging from 3 months to 8 months depending on response to therapy, under liver function test monitoring every 2 months. Two patients received intravenous amphotericin B; however, treatment had to be discontinued in both due to intolerable side-effects of the drug in one patient and treatment denial in the other patient. We found good response with resolution of proptosis in 14 patients [Fig. 5] and relief from pain in 18 patients.
Fig. 2 Computed tomography images. a Right inferior orbital hyperdense mass lesion involving the inferior rectus and inferior oblique muscles with bony erosion. b Involvement of the right orbital apex by the inflammatory process. c Bony erosion of orbital floor. d Pansinusitis with orbital extension of the inflammation

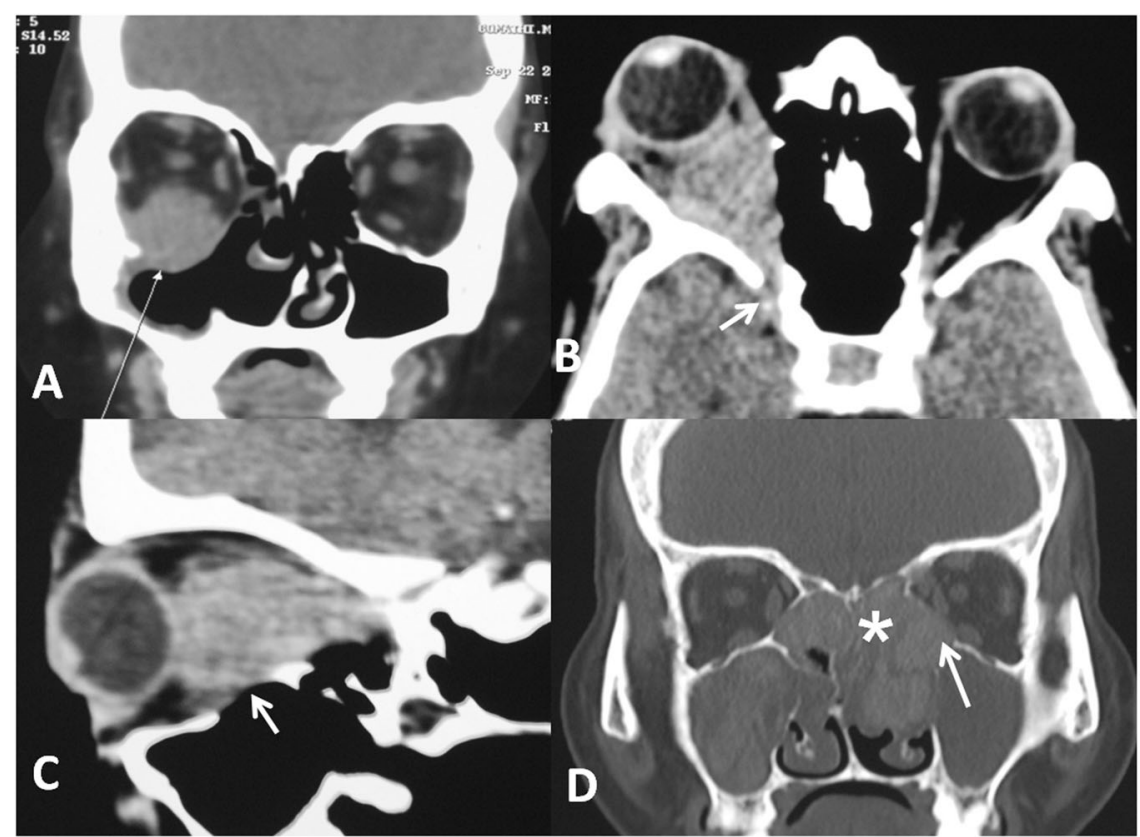


Fig. 3 Histopathological photomicrograph showing: giant cell granulomas, fibrosis, and chronic inflammatory infiltrate composed of eosinophils, lymphocytes, and plasma cells (H \& E stain). b Septate hyphae seen on GMS suggestive of aspergillosis a non-caseating multinucleate

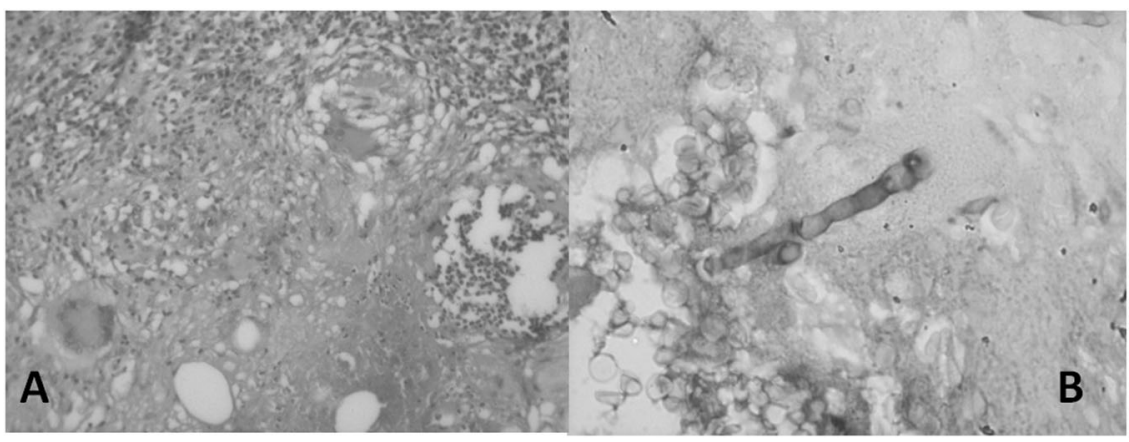

Fig. 4 Histopathological photomicrograph showing: a intravascular invasion of the fungi with formation of localized thrombus $(\mathrm{H} \& \mathrm{E}$ stain) b flat, elongated, ribbon like, collapsed aseptate fungal hyphae seen on GMS suggestive of Mucor

Fig. 5 External photograph of the patient showing a proptosis of right eye with inferior dystopia with mature cataract. b After treatment, same patient showing good resolution of proptosis with small hyperopia in right

\section{Surgical interventions}

Surgical debridement either diagnostic or debulking was performed in all patients. Patients with inferomedial mass lesions underwent endoscopic debridement by an otorhinolarygologist. Ethmoidectomy was performed in all these patients. Two patients underwent maxillectomy along with inferior orbitotomy for debulking of orbital and maxillary components. One of them developed non healing defect in the maxillary area requiring recurrent suturing and ultimately led to a depressed malar scar with lower lid ectropion. Exenteration was performed in 2 cases with non resolving disease.

\section{Visual outcome}

Four patients presented to us with decreased visual acuity and further visual compromise during the course of treatment was seen in 8 patients. (Supplemental table 1) Involvement of the orbital apex with secondary compromise of the vascular supply to the optic nerve and/or retina is the most common cause loss of vision in our series. In one patient with massive proptosis and exposure keratopathy developed secondary bacterial keratitis progressing to panophthalmitis. The eye had to be eviscerated to control the infection.

\section{Discussion}

Invasive fungal infections of the orbit in healthy patients are relatively under-recognized clinical entity. There have been cases reported from tropical countries like Sudan and India [2-6]. Tropical climate favorable for fungal growth and larger number of fungal spores in the environment may lead to a greater exposure to fungal antigens, increasing the risk of invasive sino-orbital disease even in healthy patients. All 
patients in our study were immunocompetent and majority in the young productive age group. This is similar to the mean age reported in 2 other studies from India $[3,5,6]$. Some authors consider advanced age as a predisposing risk factor and previous studies have reported this disease in patients aged more than 60 years $[7,8]$.

As reported in the literature, proptosis was the most common clinical manifestation in our series $[1,6,9,10]$. Four patients had acute onset proptosis associated with pain, restriction of extraocular movements, and decrease in vision, and 3 of these were initially diagnosed and treated as bacterial orbital cellulitis. Mauriello et al, have described 3 immunocompetent patients with sino-orbital aspergillosis presenting with an acute onset of proptosis and loss of vision. 2 patients in our series presented with periocular discharging sinuses [7]. This feature, which represents the chronicity of the disease, has not been so far reported in previously published reports.

Orbital aspergillosis has been misdiagnosed as malignancy [7, 11], temporal arteritis [7, 12], optic neuritis [13], idiopathic orbital inflammatory syndrome [12, 14], orbital apex syndrome $[8,15]$, and typical bacterial cellulitis/orbital abscess [14]. Orbital idiopathic orbital inflammatory syndrome was diagnosed in 8 out 20 cases before they presented to us. All of these were treated with systemic steroids with worsening of symptoms before they presented to our clinic. Low index of suspicion with absence of sinus related symptoms leads to diagnostic dilemmas in such cases.

Imaging features of sino-orbital aspergillosis are nonspecific and may be confused with varied orbital pathologies; most commonly idiopathic orbital inflammatory disease. Computed tomography scan usually reveals a heterogeneous, enhancing mass with density similar to extraocular muscles [16]. Involvement of paranasal sinuses usually is a helpful pointer to the diagnosis, although it may be absent in certain cases. Concomitant sinus disease has been reported in $60-90 \%$ of patients by various authors $[4,9,17]$. In our study, pansinusitis was present in $70 \%$ of patients (14 out of 20). Bone erosion is not mandatory for extension of aspergillosis and may or may not be seen [18]. However, in our study, bony erosion was seen in 50\% of the patients. Magnetic resonance imaging provides better details of the posterior orbit, optic canal, and cavernous sinus [19]. Subtle signs of sinus involvement, such as enhancement of the sinus lining and focal hypointense areas within it, may be picked up on MRI [20]. However, cost is a limiting factor for its use in developing countries.

Several authors have reported a failure to identify fungus on initial orbital biopsies [2, 14]. In the current study, incision biopsy was done to confirm the diagnosis in 19 patients; of which 4 patients underwent endoscopic biopsy while 15 patients had open biopsy. Inferolateral orbitotomy with swinging eyelid incision was the most preferred approach in our series. Repeat biopsy was performed in 3 patients as the first biopsy failed to identify the fungal elements despite high clinical suspicion. Dhiwakar et al, reported a sensitivity of $33 \%$ for incisional biopsy procedures from the paranasal sinuses [3]. Pushker et al, have suggested that FNAB may be tried in patients with sino-orbital masses involving the posterior orbit, in whom biopsy from the involved paranasal sinus is negative and incisional biopsy from the orbital mass is difficult, with high suspicion of aspergillosis [5]. They have reported positive fine needle aspiration biopsy (FNAB) in one patient with negative incision biopsy. Kuruba et al, have reported the efficacy of fine needle aspiration cytology in making an early definitive diagnosis of fungal infection and thus obviating the need for biopsy in 2 patients with sino-orbital fungal disease [21]. However, the role of FNAB remains unclear in the diagnosis of orbital aspergillosis.

It is important to distinguish allergic aspergillosis on histopathology, characterized by the presence of mucin that is rich in eosinophils and Charcot-Leyden crystals, and harbors Aspergillus hyphae with no tissue invasion [22]. Presence of tissue invasion, especially angio-invasion, which is a property of these fungi helps to clinch the diagnosis. Mucor infections are associated with a severe acute disease with relentless progression. Mucormycosis has been reported in immunocompromised patients, particularly those with severe neutropenia; however it was seen in 2 patients in our series. Recently, Rahman et al. have described mucormycosis in a non-immunocompromised patient; however, patient succumbed to death despite aggressive surgical intervention and antifungal therapy with amphotericin B. This case highlights relentless progression with poor prognosis of mucor [23].

The lack of standard treatment guidelines makes the management of invasive sino-orbital aspergillosis challenging. Aspergillus is angioinvasive and causes necrosis by infarction and direct invasion creating an anaerobic environment which is thought to support its growth and hamper drug penetration. Therefore, surgical debridement is only a plausible approach. In orbit, however, surgical debridement is limited by (1) involvement of bone and blood vessels, through which the infection has extended into the orbit; (2) presence of vital structures; and (3) an inability to be sure of the extent of disease [20]. Exenteration has been recommended by some authors in all patients with retrobulbar and apical involvement [3, 7]. Although exenteration is a potentially disfiguring treatment, it does not guarantee eradication of apical orbital disease. One patient with fungal corneal ulcer due to mucormycosis, who underwent evisceration for fungal panophthalmitis presented with residual apical mass and orbital inflammation. He was diagnosed as invasive fungal endophthalmitis and underwent exenteration. However he had persistent apical lesion on orbital 
imaging, which cleared after 4 weeks of antifungal therapy. Certain surgeons favor globe-conserving debulking combined with antifungal therapy [15]. Panda et al, reported a good response to conservative treatment with amphotericin $\mathrm{B}$ and itraconazole in immunocompetent patients with sinoorbital/sino-orbito-cerebral invasive aspergillosis [24].

Pushker et al, reported resolution of retrobulbar and apical orbital disease with antifungal therapy alone and have questioned the role of surgery in treatment of orbital aspergillosis [5]. In our experience, limited orbital debulking preserving the vital structures along with oral itraconazole has good outcomes in terms of relief of pain, resolution of proptosis and salvage of the globe. However, vision could not be salvaged in $40 \%$ of our patients. 5 of them were unable to perceive light at the last follow- up. Central retinal occlusion and optic neuropathy were the causes of visual compromise due to vascular compromise causing ischemia. The angioinvasive nature of these fungi gives rise to a localized procoagulant state with formation of thrombus within the vessel lumen which may get dislodged. This along with raised intraorbital pressure leads to vascular occlusions. Mody et al, have reported favorable visual outcomes in $62.5 \%$ of cases in their series [6].

Although, amphotericin B is considered the gold standard for the treatment of orbital aspergillosis, its adverse effects are a major deterrent for patient compliance. Less toxic liposomal drugs delivery systems are available but these are very expensive and were not affordable to most of our patients. Most authors recommend initial treatment with high doses of amphotericin B followed by a long duration of itraconazole therapy. We found good response with itraconazole in most patients in our study. We started all patients on $100 \mathrm{mg}$ twice daily and reassessed response to treatment every month. An alteration in liver enzymes is a known side effect of this drug and needs regular monitoring. At the end of 6 months, resolution of symptoms was achieved in $80 \%$ cases in our study.

In recent studies, voriconazole has been used with reported success in cases of sino-orbital aspergillosis [25]. However, the high cost of this drug is a limiting factor for long-term use especially in the rural low income population group like ours. The combination of rifampin and 5-flourocytosine has been reported to be better than single drug therapy [11].

\section{Conclusion}

In conclusion, this is a large case series of orbital fungal infections reported in healthy individuals. Relative rarity of the disease and atypical presentations masquerading varied orbital conditions, often leads to delay in diagnosis and starting therapy. Though MRI is more sensitive in picking up orbital soft tissue inflammations, CT scan remains the primary imaging modality of choice in developing countries. We recommend early surgical debulking, especially in cases involving the posterior orbit and the orbital apex, along with prolonged antifungal therapy. We found oral itraconazloe as an effective alternative in our patients. With early diagnosis and prompt therapy, visual outcomes can be improved and globe salvage is possible in majority of these patients.

\section{Summary}

\section{What was known before}

- Invasive sino-orbital fungal infections in immunocompetent patients are a rare clinical entity. Currently, there are no standard guidelines for management of these cases and the outcome is invariably poor.

\section{What this study adds}

- We present a large case series of invasive orbital fungal granulomas in immunocompetent patients in a developing country. We believe combined approach of debulking along with prolonged antifungal therapy seems to be effective in controlling orbital infection.

Author contributions Concepts, design and guarantor of the article: NGA, NV, and UK; definition of intellectual content and manuscript editing: NGA, SR, NV, and UK; literature search and clinical studies: NGA; data acquisition and data analysis: NGA, SR, and NV; manuscript preparation: NGA, SR, and UK; and manuscript review: NV and UK.

\section{Compliance with ethical standards}

Conflict of interest The authors declare that they have no conflict of interest.

Publisher's note: Springer Nature remains neutral with regard to jurisdictional claims in published maps and institutional affiliations.

\section{References}

1. Shamim MS, Siddiqui AA, Enam SA, Shah AA, Jooma R, Anwar S. Craniocerebral aspergillosis in immunocompetent hosts: Surgical perspective. Neurol India. 2007;55:274-81.

2. Kameswaran M, Al-Wadei A, Khurana P, Okafor BC. Rhinocerebral aspergillosis. J Laryngol Otol. 1992;106:981-5.

3. Dhiwakar M, Thakar A, Bahadur S. Invasive sino-orbital aspergillosis: surgical decisions and dilemmas. J Laryngol Otol. 2003;117:280-5.

4. Green WR, Font RL, Zimmerman LE. Aspergillosis of the orbit: report of ten cases and review of the literature. Arch Ophthalmol. 1969;82:302-13. 
5. Pushker N, Meel R, Kashyap S, Bajaj M, Sen S. Invasive Aspergillosis of orbit in immunocompetent patients: treatment and outcome. Ophthalmology. 2011;118:1886-91.

6. Mody KH, Ali MJ, Vemuganti GK, Nalamada S, Naik MN, Honavar SG. Orbital aspergillosis in immunocompetent patients. Br J Ophthalmol. 2014;0:1-6.

7. Mauriello JA Jr, Yepez N, Mostafavi R, Barofsky J, Kapila R, Baredes S, et al. Invasive rhinosino- orbital aspergillosis with precipitous visual loss. Can J Ophthalmol. 1995;30:124-30.

8. Marcet MM, Yang W, Albert DM, Salamat MS, Appen RE. Aspergillus infection of the orbital apex masquerading as TolosaHunt syndrome. Arch Ophthalmol. 2007;125:563-6.

9. Heier JS, Gardner TA, Hawes MJ, McGuire KA, Walton WT, Stock J. Proptosis as the initial presentation of fungal sinusitis in immunocompetent patients. Ophthalmology. 1995; 102:713-7.

10. Streppel M, Bachmann G, Arnold G, Damm M, Stennert E. Successful treatment of an invasive aspergillosis of the skull base and paranasal sinuses with liposomal amphotericin B and itraconazole. Ann Otol Rhinol Laryngol. 1999;108:205-7.

11. Yumoto E, Kitani S, Okamura H, Yanagihara N. Sino-orbital aspergillosis associated with total ophthalmoplegia. Laryngoscope. 1985;95:190-2.

12. Austin P, Dekker A, Kennerdell JS. Orbital aspergillosis: report of a case diagnosed by fine needle aspiration biopsy. Acta Cytol. 1983;27:166-9.

13. Spoor TC, Hartel WC, Harding S, Kocher G. Aspergillosis presenting as a corticosteroid-responsive optic neuropathy. J Clin Neuroophthalmol. 1982;2:103-7.

14. Whitehurst FO, Liston TE. Orbital aspergillosis: report of a case in a child. J Pediatr Ophthalmol Strabismus. 1981;18:50-4.
15. Slavin ML. Primary aspergillosis of the orbital apex. Arch Ophthalmol. 1991;109:1502-3.

16. Massry GG, Hornblass A, Harrison W. Itraconazole in the treatment of orbital aspergillosis. Ophthalmology. 1996;103:1467-70.

17. Hedges TR, Leung LS. Parasellar and orbital apex syndrome caused by aspergillosis. Neurology. 1976;26:117-20.

18. Patel PJ, Kolawole TM, Malabarey TM, Hulailah A, Hamid F, Chakaki M. CT findings in paranasal sinus aspergillosis. Clin Radiol. 1992;45:319-21.

19. Dyken ME, Biller J, Yuh WT, Fincham R, Moore SA, Justin E. Carotid-cavernous sinus thrombosis caused by Aspergillus fumigatus: magnetic resonance imaging with pathologic correlation-a case report. Angiology. 1990;41:652-7.

20. Sivak-Callcott JA, Livesley N, Nugent RA, Rasmussen SL, Saeed P, Rootman J. Localized invasive sino-orbital aspergillosis: characteristic features. Br J Ophthalmol. 2004;88:681-7.

21. Kuruba SL, Prabhakaran VC, Nagarajappa AH, Biligi DS. Orbital aspergillus infection diagnosed by FNAC. Diagn Cytopathol. 2011;39:523-6.

22. Das A, Bal A, Chakrabarti A, Panda N, Joshi K. Spectrum of fungal rhinosinusitis; histopathologist's perspective. Histopathology. 2009;54:854-9.

23. Rahman A, Akter K, Hossain S, Rashid HU. Rhino-orbital mucourmycosis in a non-immunocompromised patient. BMJ Case Rep. 2013;2013:bcr2012007863.

24. Panda NK, Saravanan K, Chakrabarti A. Combination antifungal therapy for invasive aspergillosis: can it replace high-risk surgery at the skull base? Am J Otolaryngol. 2008;29:24-30.

25. Sugai A, Oyake M, Umeda M, Umeda Y, Fujita N. Case of orbital apex syndrome caused by invasive aspergillosis successfully treated during the diagnostic procedure by the use of voriconazole. Rinsho Shinkeigaku. 2008;48:746-9. 